\title{
Variability in vancomycin levels in an intensive care population explained by variability in clearance
}

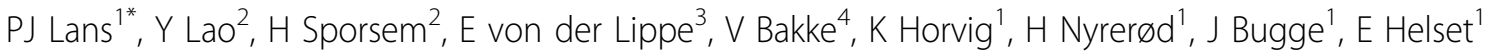 \\ From ESICM LIVES 2015 \\ Berlin, Germany. 3-7 October 2015
}

\section{Introduction}

Vancomycin levels are frequently subtherapeutic or too high in critically ill patents.

\section{Objectives}

To study how variability in clearance: creatinine clearance $(\mathrm{CrCL})$, total vancomycin clearance (totVCL) and extracorporal clearance (CRRTCL) correlated with vancomycin through levels.

\section{Methods}

In a prospective observational study we included patients started on vancomycin, from four different intensive care units (ICU) at Oslo University Hospital. Blood samples as well as ultrafiltrate samples from patients on continuous renal replacement therapy (CRRT), were collected in one dosage interval on three consecutive days 24 hours(hrs) from start. The observation time was 72 hrs. Vancomycin concentrations were determined using a commercial assay (Cobas C Systems Roche). CrCL, totVCL and CRRTCL were calculated for each dosage interval.

\section{Results}

76 patients were included, 48 non-CRRT and 28 CRRT: 15 on continuous venovenous hemodialysis (CVVHD) and 13 on continuous venovenous hemofiltration $(\mathrm{CVVH})$. In the CRRT group the effluent flow rate was $2099 \pm 435 \mathrm{ml} / \mathrm{hr}$ (SD). Age mean was $50.8 \pm 15.1$ years, $74 \%$ men and $26 \%$ women. SAPS mean was $47.2 \pm 16.0$. The reasons for admittance to the ICU were trauma $25 \%$, postoperative complications $42 \%$, septicaemia $15 \%$ and respiratory failure $18 \%$. The average vancomycindose at the points of measurements was $1207,4 \pm 550,4 \mathrm{mg}$. In the non-CRRT group the mean $\mathrm{CrCL}$ was $117 \pm 9 \mathrm{ml} / \mathrm{min}(\mathrm{SEM})$, and the totVCL was $88 \pm 6 \mathrm{ml} / \mathrm{min}$. There were no significant differences between the measurements at 24,48 and $72 \mathrm{hrs}$. A significant correlation was found between CrCL and totVCL in the non-CRRT group as expected. In the CRRT group the mean totVCL was $51 \pm 2 \mathrm{ml} / \mathrm{min}$, a significant reduction in clearance with $43 \%$ as compared to the non-CRRT group. The mean CRRTCL was $26 \pm$ $3 \mathrm{ml} / \mathrm{min}$. No significant differences was observed between the sieving coefficients in CVVH $(0.71 \pm 0.001)$ compared to CVVHD $(0.69 \pm 0.02)$. There was a significant negative correlation between totVCL and vancomycin through concentrations in both the non-CRRT and the CRRT group, but a stronger negative correlation in the non-CRRT group $(\mathrm{p}<0.001)$.

\section{Conclusions}

In the CRRT group, clearance of vancomycin by CRRT contributed to $50 \%$ of totVCL independent of CRRT mode. The reduced totVCL in patients on CRRT, should lead to reduced maintenance dose of vancomycin to avoid to high through concentrations. Measurement of $\mathrm{CrCL}$ may be useful in non-CRRT critically ill patients to avoid subtherapeutic vancomycin levels.

\section{Authors' details}

${ }^{1}$ Oslo University Hospital, Department of Anesthesiology, Oslo, Norway. ${ }^{2}$ Oslo Hospital Pharmacy, Oslo, Norway. ${ }^{3}$ Oslo University Hospital, Department of Infectious Diseases, Oslo, Norway. ${ }^{4}$ Oslo University Hospital, Medical School, Oslo, Norway.

Published: 1 October 2015

\section{doi:10.1186/2197-425X-3-S1-A629}

Cite this article as: Lans et al:: Variability in vancomycin levels in an intensive care population explained by variability in clearance. Intensive Care Medicine Experimental 2015 3(Suppl 1):A629. 